\title{
Pulmonary tumor thrombotic microangiopathy successfully treated with corticosteroids: a case report
}

\author{
Shinichi Miyazaki ${ }^{1 *}$, Takuya Ikeda', Genshi Ito' ${ }^{1}$ Masahide Inoue ${ }^{1}$, Keiji Nara², Yuko Nishinaga ${ }^{3}$ \\ and Yoshinori Hasegawa ${ }^{3}$
}

\begin{abstract}
Background: Pulmonary tumor thrombotic microangiopathy is a special type of tumor thromboembolism. We report the case of a patient who developed pulmonary tumor thrombotic microangiopathy with alveolar hemorrhage. Almost all patients with pulmonary tumor thrombotic microangiopathy die within 1 week of the onset of dyspnea; however, the prognosis in this case was better, with 10 weeks of survival from presentation.

Case presentation: A 62-year-old Japanese man was referred to our hospital with a 4-week history of dyspnea on exertion and severe pulmonary hypertension. Five years previously, he had undergone distal gastrectomy for gastric cancer. He was afebrile, normotensive, and hypoxemic. A physical examination was unremarkable except for purpura on his upper extremities and trunk. Blood tests showed anemia and disseminated intravascular coagulation. Chest computed tomography revealed diffuse ground-glass opacities with emphysema in his upper lungs, moderate pleural effusions, mediastinal lymphadenopathy, and enlargement of the right ventricle and main pulmonary artery. A computed tomography pulmonary angiogram showed no evidence of pulmonary embolism. Lung perfusion scintigraphy showed multiple segmental defects. Although recurrence of gastric cancer was confirmed from the results of bone marrow biopsy, bronchoscopy was not performed due to bleeding diathesis. He was treated with corticosteroids, antibiotics, and platelet transfusion, following which resolution of the abnormal lung shadows and right ventricular pressure overload along with partial alleviation of respiratory failure was observed. Because of his poor performance status, he was eventually transited to palliative care and died 6 weeks after admission. Necropsy of the lung confirmed the diagnosis of pulmonary tumor thrombotic microangiopathy with alveolar hemorrhage.

Conclusions: Pulmonary tumor thrombotic microangiopathy should be considered in the differential diagnosis of patients with cancer who present with severe pulmonary hypertension. In pulmonary tumor thrombotic microangiopathy, local inflammation in pulmonary microvasculature may contribute to pulmonary hypertension, and regulation of inflammation using corticosteroids may help improve the prognosis.
\end{abstract}

Keywords: Pulmonary embolism, Pulmonary hypertension, Rare lung diseases

\section{Background}

Pulmonary tumor thrombotic microangiopathy (PTTM) is a special type of tumor thromboembolism. The condition is histologically characterized by microscopic tumor emboli with occlusive fibrointimal proliferation in the pulmonary microcirculation. It should be considered in the differential diagnosis of pulmonary hypertension, particularly in patients with known cancer. We report the case of a patient who developed PTTM with alveolar hemorrhage. Almost all patients with PTTM die within 1 week of the onset of dyspnea; however, the prognosis in this case was better, with 10 weeks of survival from the day of presentation.

\footnotetext{
* Correspondence: miyazaki.sin1@gmail.com

'Department of Respiratory Medicine, Yokkaichi Municipal Hospital,

Sibata2-2-37, Shibata, Yokkaichi-shi, Mie 510-0822, Japan

Full list of author information is available at the end of the article
}

\section{Case presentation}

A 62-year-old Japanese man presented with a 4-week history of dyspnea on exertion. There was no history of 
fever, anorexia, cough, or chest pain. He had visited another hospital, where he was found to be hypoxemic; a chest X-ray showed consolidation in his upper lungs (Fig. 1a). Echocardiography revealed marked right ventricular dilation and flattening of the interventricular septum with severe pulmonary hypertension (transtricuspid pressure gradient, $109 \mathrm{~mm} \mathrm{Hg}$ ). He was referred to our hospital within a few hours of presentation. Five years previously, he was diagnosed as having gastric cancer (pT2N2M0 stage IIIA), for which he underwent distal gastrectomy and adjuvant chemotherapy with tegafur plus uracil. On follow-up, after completion of 2 years of chemotherapy, no recurrence was observed. His family history was unremarkable. He was a former tobacco smoker with 20 pack-years of smoking history; there was no history of alcohol intake.

$\mathrm{He}$ was afebrile, normotensive, and hypoxemic: blood oxygen saturation $\left(\mathrm{SpO}_{2}\right)$ 88\%, $3 \mathrm{~L}$ oxygen by nasal cannula. A physical examination was unremarkable with the exception of purpura on his upper extremities and trunk. Blood tests showed normocytic anemia with leukoerythroblastosis and disseminated intravascular coagulation. His serum alkaline phosphatase level was remarkably elevated (3205 U/L), but serum transaminase level was normal. Chest computed tomography (CT) revealed diffuse ground-glass opacities with emphysema in his upper lungs, moderate pleural effusions, mediastinal lymphadenopathy, and enlargement of the right ventricle and main pulmonary artery (Fig. 2a). A CT pulmonary angiogram (CTPA) showed no evidence of pulmonary embolism. Lung perfusion scintigraphy showed multiple segmental defects (Fig. 3a); bone scintigraphy showed diffuse uptake (Fig. 3b). Although recurrence of gastric cancer was confirmed from the results of a bone marrow biopsy (Fig. 3c), bronchoscopy was not performed due to bleeding diathesis.
In a patient with cancer presenting with subacute dyspnea, severe pulmonary hypertension and negative pulmonary angiogram are consistent with a diagnosis of PTTM. Differential diagnosis of diffuse ground-glass opacities in the upper lungs includes community-acquired pneumonia, interstitial pneumonia, and alveolar hemorrhage.

He was treated with corticosteroids (prednisone $40 \mathrm{mg}$ daily), intravenously administered antibiotics (cefepime), and platelet transfusion (three units of whole blood-derived platelets to maintain a platelet count of $50,000 / \mu \mathrm{L}$ ). Following treatment, resolution of the abnormal lung shadows was observed along with improvement in right ventricular pressure overload (Figs. $1 \mathrm{~b}$ and $2 \mathrm{~b}$ ), and partial alleviation of respiratory failure $\left(\mathrm{SpO}_{2} 96 \%, 2 \mathrm{~L}\right.$ oxygen by nasal cannula). Plans for chemotherapy were deferred because of his poor performance status, and he was eventually transited to palliative care. Favorable conditions continued for several weeks. On day 41 of hospitalization, he developed fever with rigors, and empirical antibiotic treatment (cefepime and vancomycin) was initiated. He soon turned afebrile; however, on day 45 of hospitalization, he suddenly died of respiratory failure. Necropsy of the lung confirmed the diagnosis of PTTM (Fig. 4); there was no evidence of pulmonary embolism. Alveolar hemorrhage with abundant hemosiderin-laden histiocytes was observed.

\section{Discussion}

Our patient was a 62-year-old Japanese man with a 4-week history of dyspnea on exertion. Key findings included hypoxemia, severe pulmonary hypertension, and negative pulmonary angiogram. Recurrence of gastric cancer was confirmed from the results of a bone marrow biopsy; PTTM was considered. He was treated with conservative management, following partial alleviation of respiratory failure, and died 6 weeks after admission. Most patients with PTTM die within a week of dyspnea onset; however, our patient survived for 10 weeks from the day of presentation.

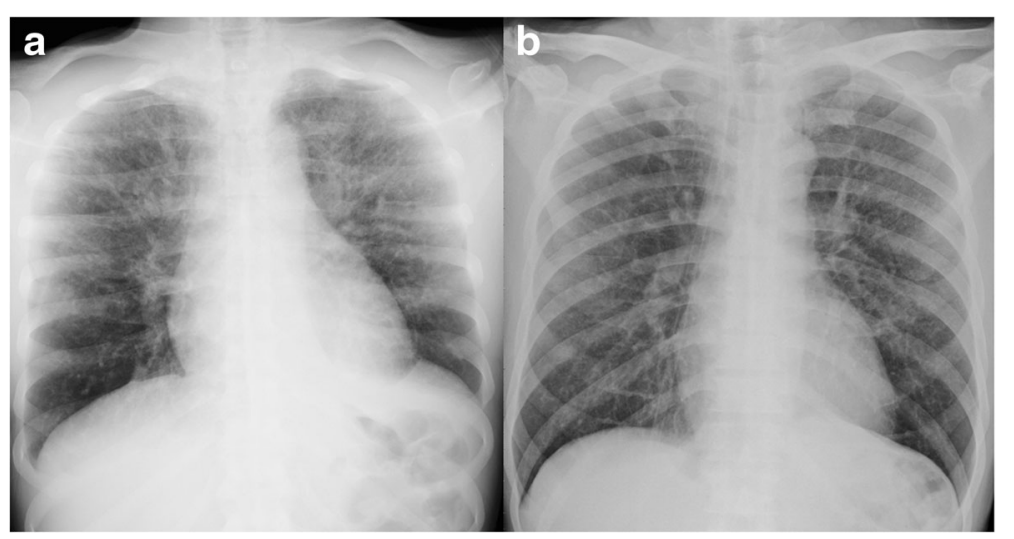

Fig. 1 Chest X-ray. Chest X-ray at admission showing consolidation in the upper lungs (a); the abnormal shadow disappeared 4 weeks after admission (b) 


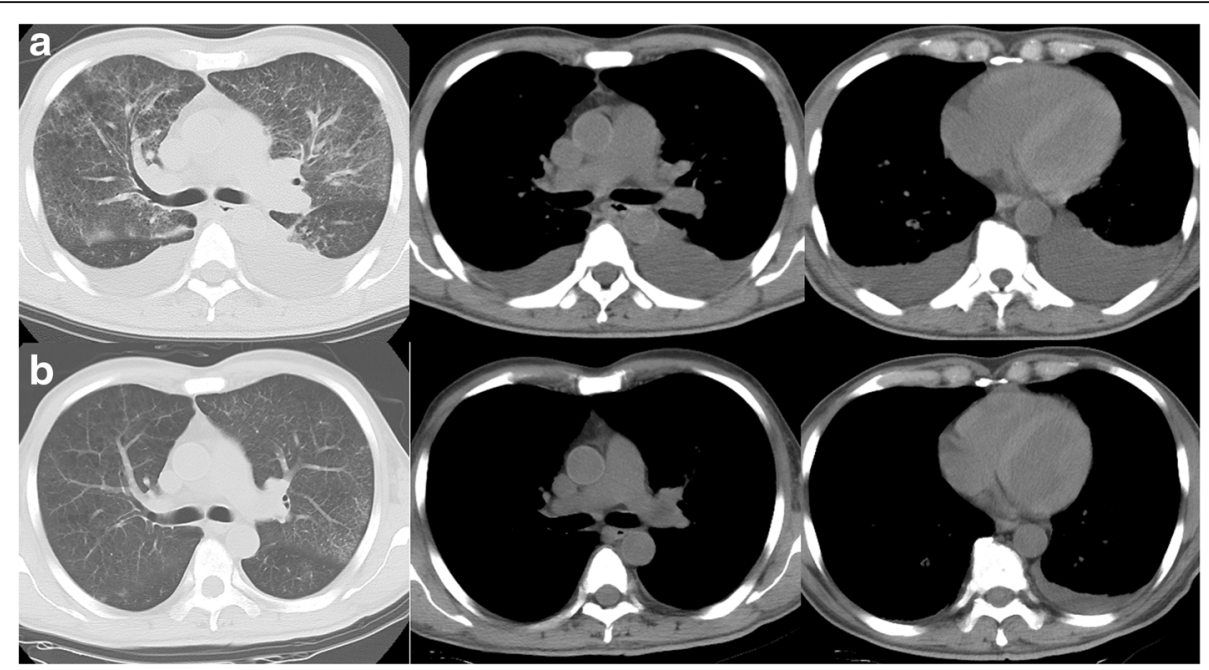

Fig. 2 Computed tomography of the chest. a Computed tomography radiograph showing ground-glass opacities in the upper lungs with moderate pleural effusions, and findings suggestive of right ventricular pressure overload (enlargement of the right ventricle and main pulmonary artery). $\mathbf{b}$ Computed tomography radiograph at 4 weeks after admission showing resolution of the abnormal lung shadows along with signs of alleviation of the right ventricular pressure overload. These helical computed tomography images consisted of 5-mm collimation sections, with window settings appropriate for viewing both the lung (window level from $-700 \mathrm{HU}$, window width from $2000 \mathrm{HU}$ ) and the mediastinum (window level from $40 \mathrm{HU}$, window width from $400 \mathrm{HU}$ )

PTTM, a special type of tumor thromboembolism, is found in $3.3 \%$ of postmortem cases of carcinoma; of all PTTM cases, $52 \%$ are attributable to gastric cancer (especially mucinous or signet ring adenocarcinoma) [1]. Clinical manifestations include progressive dyspnea, cough, hypoxia, and pulmonary hypertension. Chest radiography is typically normal; reported chest CT findings include beading of peripheral subsegmental arteries, interlobular septal thickening [2], and tree-in-bud pattern [3]. Almost all patients with PTTM die within 1 week of the onset of dyspnea [4], and establishing an antemortem diagnosis is a challenge. The rapid progression usually prevents treatment targeting the primary tumor. The prognosis in this case was better than that

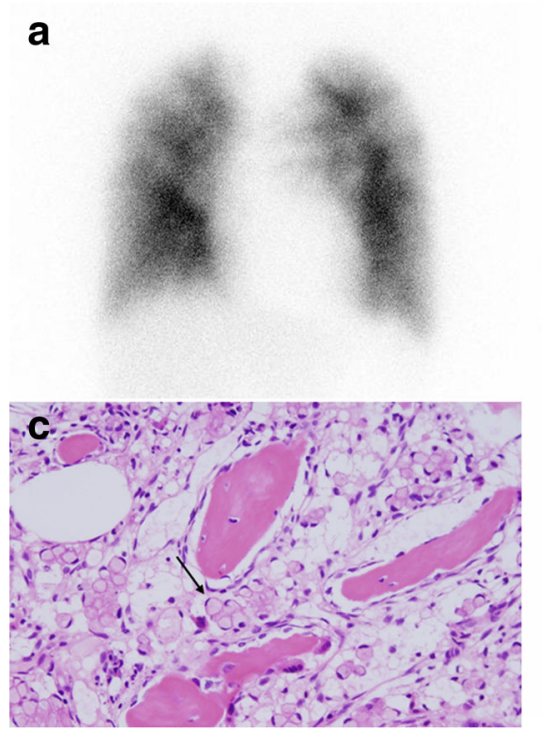

\section{b}

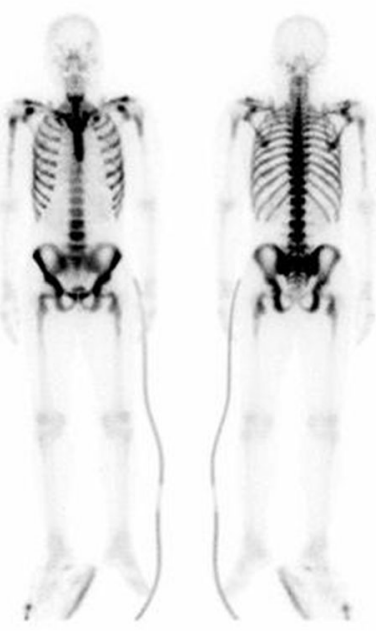

Fig. 3 Lung perfusion scintigraphy, bone scintigraphy, and bone marrow biopsy. a Lung perfusion scintigraphy showing multiple segmental defects. b Bone scintigraphy showing diffuse uptake. c Signet ring cells (arrow) in bone marrow (hematoxylin and eosin, $\times 100$ ) 


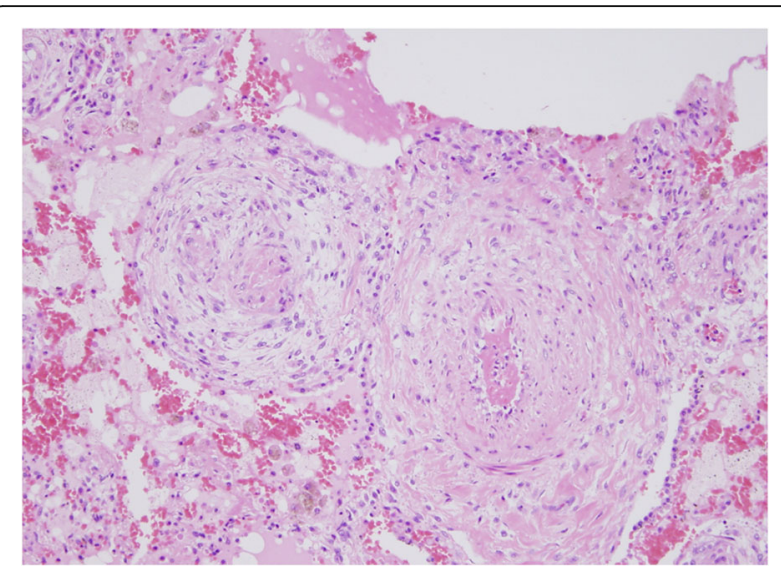

Fig. 4 Necropsy of the lung. Obliterated small vessels with fibrointimal proliferation (hematoxylin and eosin, $\times 100$ )

in the previous reports. In PTTM, tumor emboli in pulmonary vessels may not only damage the endothelium but also activate the coagulation systems and release inflammatory mediators and growth factors, including tissue factor, vascular endothelial growth factor (VEGF) [5], platelet-derived growth factor (PDGF) [6], and osteopontin [7], which induce fibrocellular intimal proliferation. PDGF induces macrophage recruitment and VEGF upregulation in cancer cells [8], which leads to inflammation through angiogenesis and enhanced vascular permeability [9]. Osteopontin not only regulates macrophage recruitment but also modulates the production of pro-inflammatory cytokines by macrophages [10]. Several reports have demonstrated that the cytokines interleukin (IL)-1 $\beta$, IL-6, and tumor necrosis factor- $\alpha$, released by activated macrophages, play an important role in the pathogenesis of pulmonary arterial hypertension (PAH) [11]. Moreover, inflammatory stimuli including that from IL-1 $\beta$ have been shown to exacerbate hypoxic vasoconstriction in a mouse model of PAH [12]. In PTTM, corticosteroid therapy may help control local inflammation in the pulmonary microvasculature and improve hemodynamics by alleviation of pulmonary vasoconstriction [13]. Our experience with this case suggests that the control of local inflammation with corticosteroid therapy may have helped improve the prognosis, although the time from the onset of pulmonary symptoms to death ranged from 1 day to 12 weeks in one series [14].

\section{Conclusions}

PTTM should be considered in the differential diagnosis of patients with cancer who present with severe pulmonary hypertension. In PTTM, local inflammation in the pulmonary microvasculature may contribute to pulmonary hypertension, and control of inflammation with corticosteroids may help improve the prognosis.

\section{Abbreviations}

CT: Computed tomography; CTPA: Computed tomography pulmonary angiogram; IL: Interleukin; PAH: Pulmonary arterial hypertension; PDGF: Platelet-derived growth factor; PTTM: Pulmonary tumor thrombotic microangiopathy; $\mathrm{spO}_{2}$ : Blood oxygen saturation; VEGF: Vascular endothelial growth factor

\section{Acknowledgements}

Not applicable.

Funding

There was no funding for this case report.

\section{Availability of data and materials}

Data are available from the corresponding author on reasonable request.

\section{Authors' contributions}

SM cared for the patient, performed the clinical reasoning, and wrote the article. TI, GI, YN, and MI cared for the patient. KN made the pathological diagnosis and contributed microscopic pictures. $\mathrm{YH}$ helped in preparing this manuscript. All authors read and approved the final manuscript.

\section{Ethics approval and consent to participate}

Not applicable.

\section{Consent for publication}

Written informed consent was obtained from the patient's next of kin for publication of this case report and any accompanying images. A copy of the written consent is available for review by the Editor-in-Chief of this journal.

\section{Competing interests}

The authors declare that they have no competing interests.

\section{Publisher's Note}

Springer Nature remains neutral with regard to jurisdictional claims in published maps and institutional affiliations.

\section{Author details}

${ }^{1}$ Department of Respiratory Medicine, Yokkaichi Municipal Hospital, Sibata2-2-37, Shibata, Yokkaichi-shi, Mie 510-0822, Japan. Department of Pathology, Yokkaichi Municipal Hospital, Sibata2-2-37, Shibata, Yokkaichi-shi, Mie 510-0822, Japan. ${ }^{3}$ Department of Respiratory Medicine, Nagoya University Graduate School of Medicine, 65 Tsurumai-cho, Showa-ku, Nagoya 466-8560, Japan.

Received: 4 August 2017 Accepted: 21 November 2017

Published online: 23 December 2017

\section{References}

1. von Herbay A. Pulmonary tumor thrombotic microangiopathy with pulmonary hypertension. Cancer. 1990;66:587-92.

2. Shepard JA. Pulmonary intravascular tumor emboli: dilated and beaded peripheral pulmonary arteries at CT. Radiology. 1993;187:797-801.

3. Tack D. Tree-in-bud pattern in neoplastic pulmonary emboli. AJR. 2001;176: 1421-2.

4. Miyano S. Pulmonary tumor thrombotic microangiopathy. J Clin Oncol. 2007:25:597-9.

5. Chinen K. Pulmonary tumor thrombotic microangiopathy caused by a gastric carcinoma expressing vascular endothelial growth factor and tissue factor. Pathol Int. 2005;55:27-31.

6. Minatsuki S. Platelet-derived growth factor receptor-tyrosine kinase inhibitor imatinib, is effective for treating pulmonary hypertension induced by pulmonary tumor thrombotic microangiopathy. Int Heart J. 2015;56:245-8.

7. Takahashi F. Osteopontin expression in pulmonary tumor thrombotic microangiopathy caused by gastric carcinoma. Pathol Int. 2009;59:752-6.

8. Price LC. Pulmonary tumour thrombotic microangiopathy. Curr Opin Pulm Med. 2016;22:421-8.

9. Shaik-Dasthagirisaheb YB. Vascular endothelial growth factor (VEGF), mast cells and inflammation. Int J Immunopathol Pharmacol. 2013;26:327-35.

10. Lund SA. The role of osteopontin in inflammatory processes. J Cell Commun Signal. 2009;3:311-22. 
11. Groth A. Inflammatory cytokines in pulmonary hypertension. Respir Res. 2014;15:47.

12. Song Y. Increased susceptibility to pulmonary hypertension in heterozygous BMPR2-mutant mice. Circulation. 2005:112:553-62.

13. Higashi A. The potential role of inflammation associated with interaction between osteopontin and CD44 in a case of pulmonary tumor thrombotic microangiopathy caused by breast cancer. Intern Med. 2015;54:2877-80.

14. Shields DJ. Pulmonary hypertension attributable to neoplastic emboli: an autopsy study of 20 cases and a review of literature. Cardiovasc Pathol. 1992;1:279-87.

Submit your next manuscript to BioMed Central and we will help you at every step:

- We accept pre-submission inquiries

- Our selector tool helps you to find the most relevant journal

- We provide round the clock customer support

- Convenient online submission

- Thorough peer review

- Inclusion in PubMed and all major indexing services

- Maximum visibility for your research

Submit your manuscript at www.biomedcentral.com/submit 